\title{
Stability of Lipid Films Formed on $\gamma$-Aminopropyl Monolayers
}

Todd W. McBee and S. Scott Saavedra*

Department of Chemistry, University of Arizona, Tucson, Arizona 85721

\section{Supporting Information}
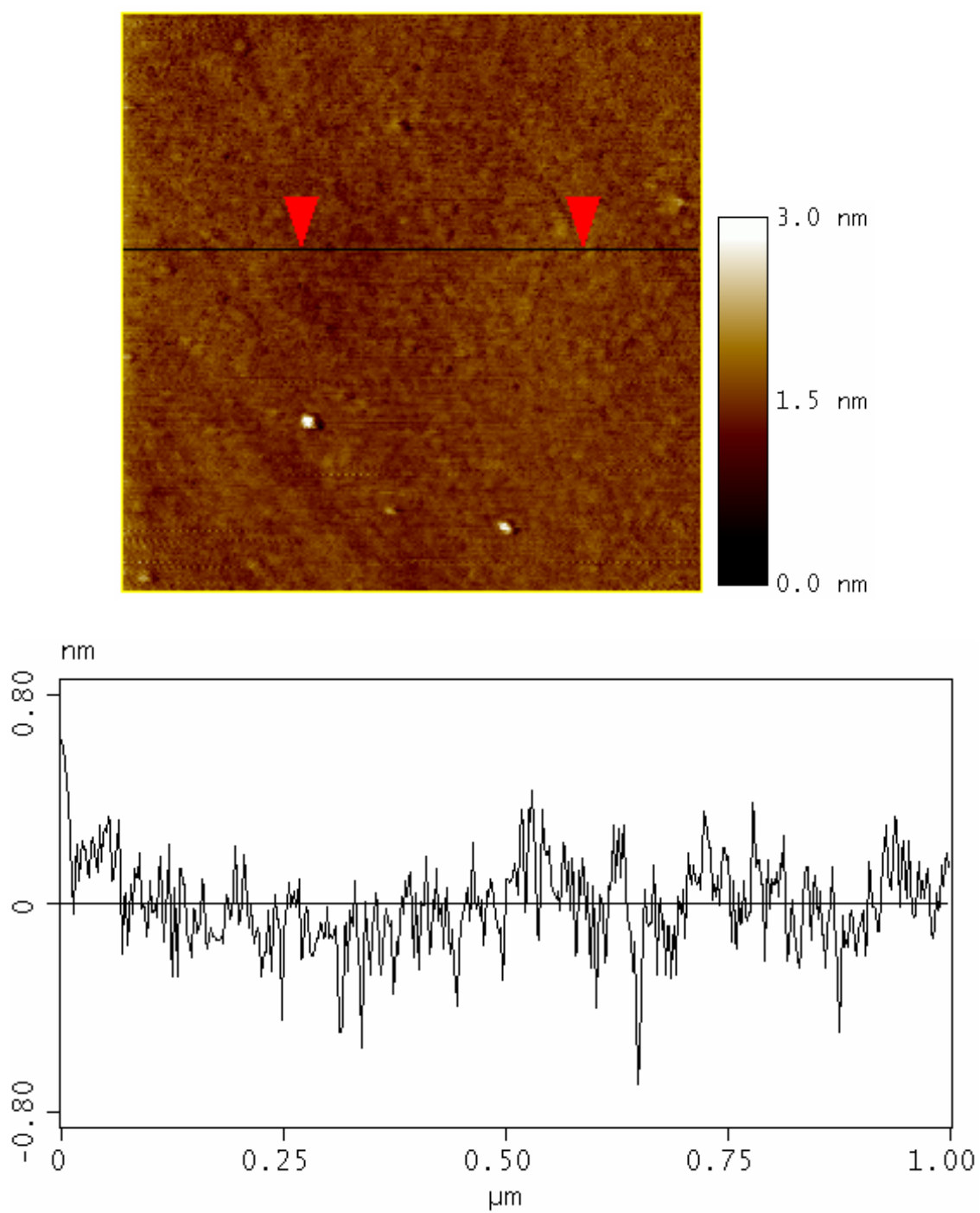

Figure S1: Representative height AFM image (1 $\mu \mathrm{m} \times 1 \mu \mathrm{m})$ and linescan of a dried GAPS-I coating on an oxidized Si wafer. The rms roughness of this surface was $0.18 \mathrm{~nm}$, which is slightly greater than that of the bare oxidized Si wafer $(0.11 \mathrm{~nm})$. The two large features seen in the lower part of the image are likely adsorbed dust particles. 

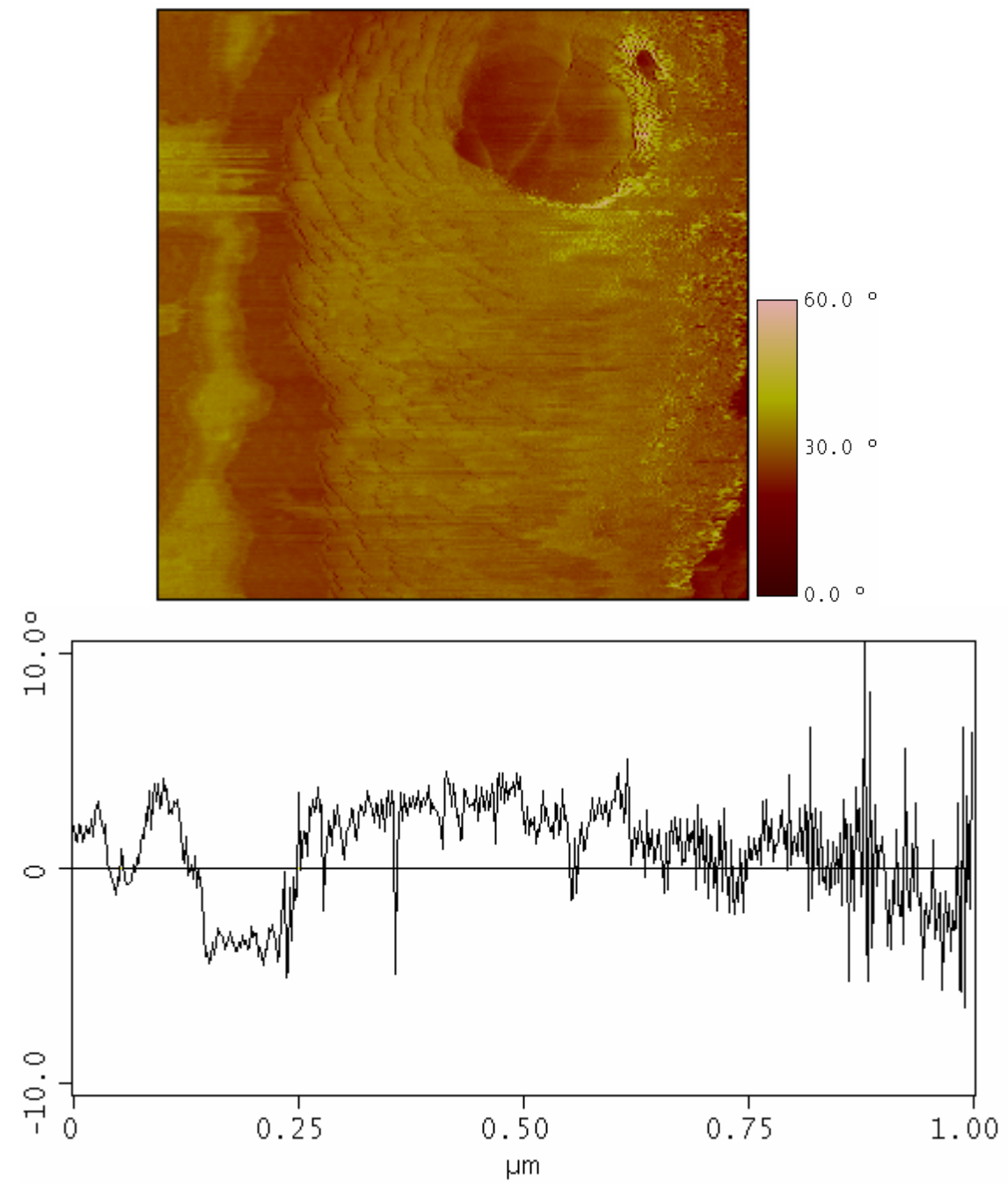

Figure S2: Phase AFM image and linescan of a SLM that was deposited on an oxidized Si wafer coated with GAPS-I and then withdrawn from water. This image was acquired simultaneously with the $1 \mu \mathrm{m} \times 1 \mu \mathrm{m}$ height image shown in Figure 2 of the manuscript. Note that the phase does not vary significantly over the length of the linescan, which indicates that the surface composition of the SLM is relatively uniform across this region. 


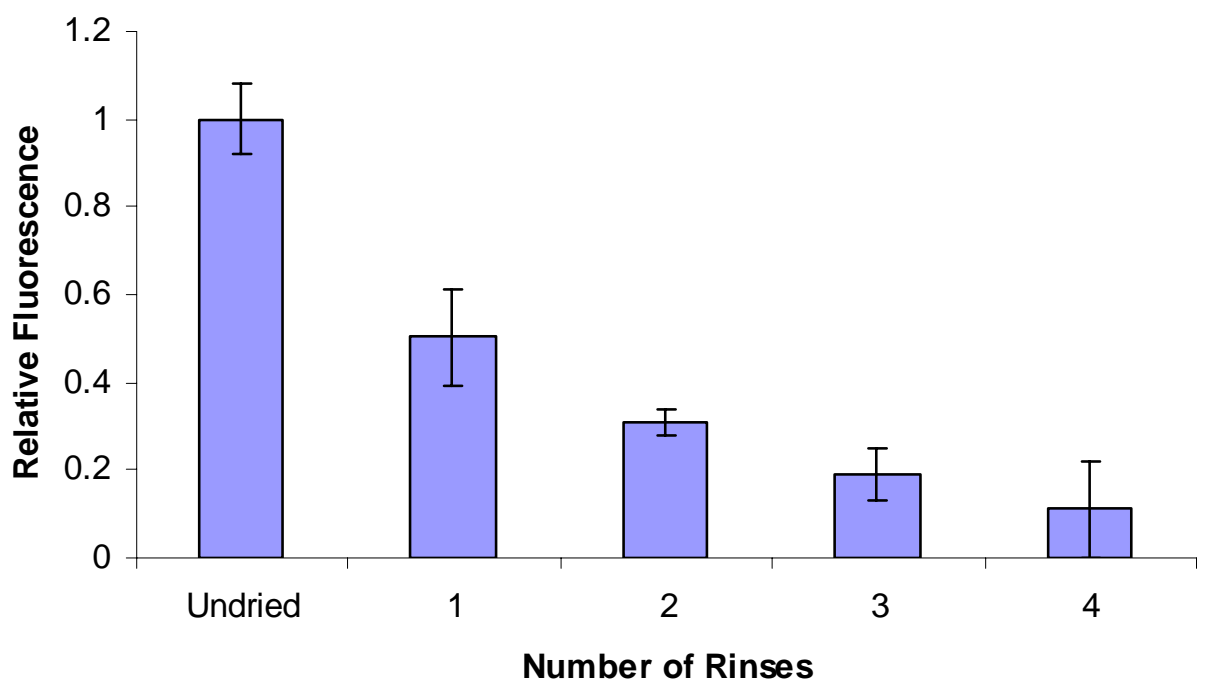

Figure S3: SLMs were formed by fusion of SUVs composed of 4:1 DPPC:DMPC, doped with 0.5\% TR-DHPE, in $20 \mathrm{mM}$ phosphate, pH 7.36, on GAPS-I slides. Fluorescence intensities were measured on hydrated SLMs as a function of the number of times that the SLM-coated substrate was withdrawn and then reimmersed in buffer. The data were normalized to the initial intensity measured after the SLM was rinsed in buffer to remove unfused vesicles, without exposing it to air. The error bars represent three measurements on independently prepared samples. 


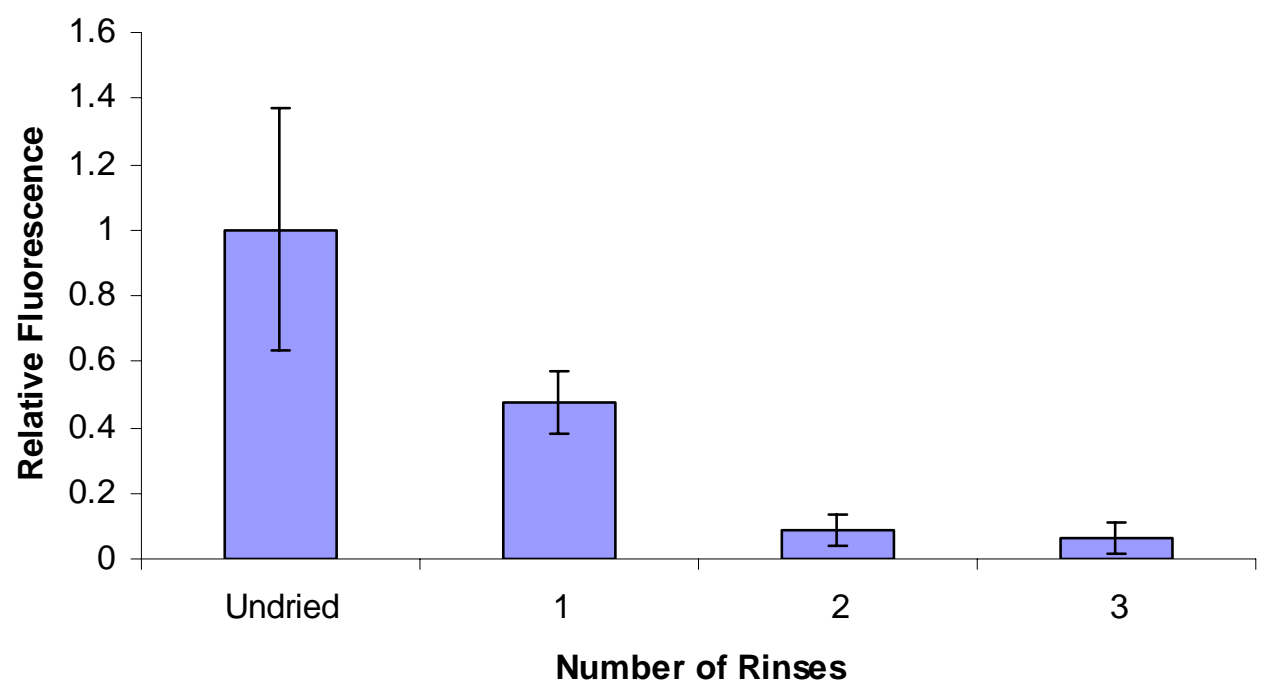

Figure S4: SLMs composed of DOPC and doped with doped with 0.5\% TR-DHPE were deposited on GAPS-II slides. Fluorescence intensities were measured on hydrated SLMs as a function of the number of times that the lipid-coated substrate was withdrawn and then reimmersed in deionized water. The data were normalized to the initial intensity measured after the SLM was rinsed to remove unfused vesicles, without exposing it to air. The error bars represent three measurements on independently prepared samples. 


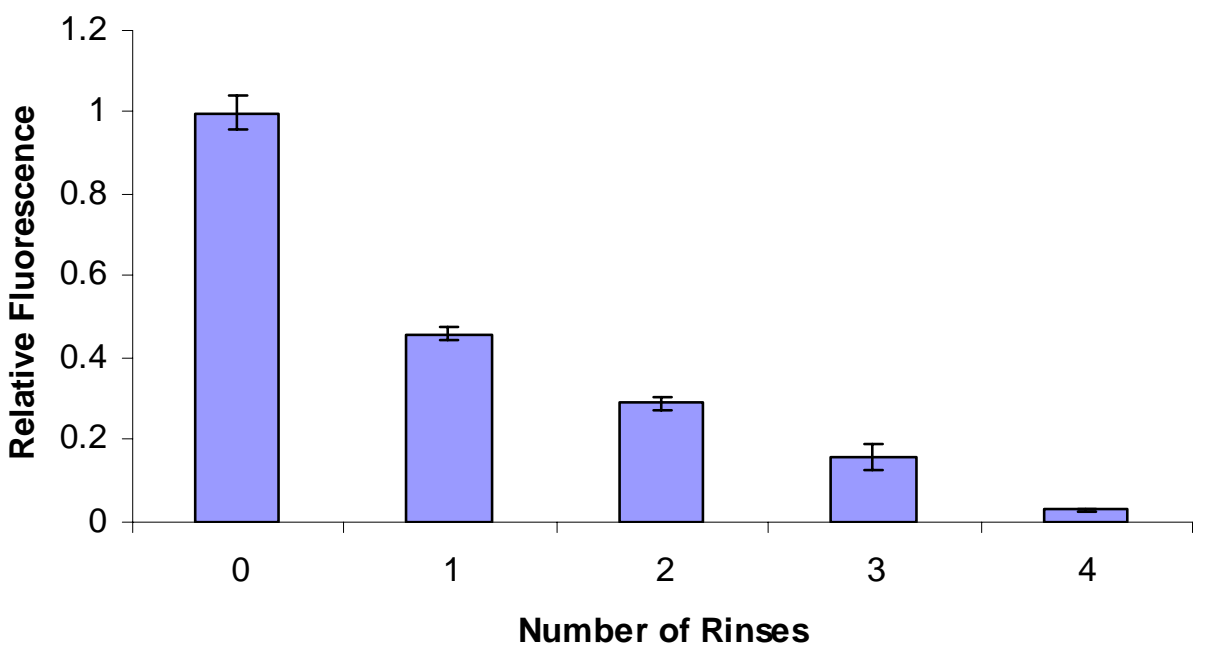

Figure S5: DPPC:DMPC (4:1) vesicles were formed in $20 \mathrm{mM}$ phosphate buffer, $\mathrm{pH} 7.36$, in the presence of 5-carboxyfluorescein (2 $\mathrm{mM}$ ). Excess (unentrapped) dye was separated from vesicles on a Sephadex G-50 column. SLMs were formed by fusion on GAPS-II slides. Fluorescence intensities were measured on hydrated SLMs as a function of the number of times that the lipidcoated substrate was withdrawn and then reimmersed in deionized water. The data were normalized to the initial intensity measured after the SLM was rinsed to remove unfused vesicles, without exposing it to air. The error bars represent three measurements on independently prepared samples. 